\title{
N82 32537
}

TDA Progress Plepon 42 10

\section{The VLSI Design of a Reed-Solomon Encoder Using Berlekamp's Bit-Serial Multiplier Algortithm}

T. K. Truong and L. J. Deutsch

Communications Systems Research Section

I. S. Reed, I.S. Hsu, K. Wang. and C.S. Yeh

University of Southern Colifornia

\begin{abstract}
E. R. Berlekamp has developed for the Jet Propulsion Labonatsy a bit-serial multiplication algorithm for the encoding of Reed Solomon (RS) codes, using a dual besis over a Galois field. The comentional RS-encoder for long codes often requires look-up tables to perform the multiplication of two field elements. Bertekamp's algorithm requires only shiffing and exchustive-OR operations. It is shown in this paper that the new duai-basis $(255.223)$ RSencoder can be realized reactily on a single VLSI chip with NMCis rechnology.
\end{abstract}

\section{Introduction}

A concatenated Reed-Solumon/Viterbi channel encoding sysiem has been suggested both by the European Space Pegency (ESA) (Ref. 1) and JPL (Refs. 2, 3) for the deep-space downlink. The standard RSencoder design developed by JPL assumes the following cedes and parameters.

Let $G F\left(2^{m}\right)$ be a finite field. Then an RS code is a sequence of the symbols in $G F\left(2^{m}\right)$. This sequence of symbols can be considered to be the coefficients of a polynomial. The code polynomial of such a code is

$$
C(x)=\sum_{i=0} c_{i} x^{i}
$$

wnere $c_{\mathrm{v}}+G F\left(2^{\mathrm{m}}\right)$
The parameters of an RS code are summarized as follows:

$$
\begin{aligned}
& m=\text { number of bits per symbol } \\
& n=2^{m}-1=\text { the length of a codeword in symbols } \\
& t=\underset{\text { rected }}{ } \\
& d=2 t+1=\text { design distance } \\
& 2 t=\text { number of check symbols } \\
& k=n-2 t=\text { number of i.fornation symbols }
\end{aligned}
$$

In the JPL design, $m=8, n=255, t=16, d=33,2 t-32$, and $k=223$. This code is the $(255,223) \mathrm{RS}$ code. 
The generator polynumial of an RS cude is defined by

$$
g(x)=\sum_{i=b}^{b+2 x-1}\left(x-\gamma^{j}\right)=\sum_{i=0}^{2 t} g_{i} x^{i}
$$

where $b$ is a nonnegative integer, usually chosen to be 1 , and $\gamma$ is a primitive element in $G F 2^{m}$ ). In order to reduce the complexity of the encoder it is desirable to make the coefficients of $g(x)$ symmetric so that $g(x)=x^{-d-1} g(1 / x)$. To accomplish this $b$ must be chosen to satisfy $2 b+d-2=2^{m}-1$. Thus for the JPL code $b=112$

Let $\zeta(x)=c_{2 \mathrm{r}} x^{2 t}+c_{2 n+1} x^{2 n+1}+\cdots+c_{n-1} x^{n-1}$ and $P(x)=$ $c_{0}+c_{1} x+\cdots+c_{2+1} x^{2 r-1}$ be the information polynomial and the check polynomial. respectively. Then the encoded RS code polynomial is represented by

$$
\text { - } \quad C(x)=I(x)+P(x)
$$

To be an RS code $C(x)$ must be also a maltiple of $g(x)$. That is.

$$
q(x)=q(x) g(x)
$$

To find $P(x)$ in Eq. (3) such that Eq. (4) is true, divide !(x) by $g(x)$. The division algonthm yields

$$
I(x)=q(x) g(x)+r(x)
$$

Also let $(x)=-P(x)$, then by Eq. (5)

$$
q(x) g(x)=I(x)-H(x)=I(x)+P(x)=Q(x)
$$

Figure 1 shows the structure of a terror cirrecting RS encoder over $G F\left(2^{m}\right)$. In Fig. I $R$, for $0<i \leqslant 2 t-I$ and $Q$ are $m$-hit registers. Initally all repusters are set to zero, and both switches (controlled by a control signal SL) are set to posi. IIIII A.

The intiomation symbols $c_{n-1}, \cdots, c_{21}$ are fed into the division curcult of the encoder and also transmitted out of the encirdet one by one The quotient coefficients are generated and loaded into $Q$ register seyuentially. The remainder coefficients are computed successively. Immediately after $c_{2 t}$ is fed to the curcuit, both switches are set to position $R$. At the very sme miment $c_{2 t-1}$ is computed and transmitted. Simulta. neously, $c$, is being computed and loaded into register $K, f$. $0<i<2 t-2$ Next $c_{2 r}, \cdot$. . in are transmitted out of the encorder one by one $c_{2+-2}, c_{0}$ retain their values because the content of $Q$ is set to zero when the upper switich is at position $B$.
The complexity of the design of an RS encoder results from the computation of protucts $88_{i}$ for $0<i<2 t-2$. Thew computations can be performed in several ways (Ref. 3). Unfortunately none of them is suited to the phpeline procescing structures usually seen in VISI design. Recenthy. Berlekunp (Ref. 4) develnped a bitserial multiplier alforitim that has the features needed to solve this problem. Pextman and Lee (Ref. 5) show in detal the mathematical basis for this algorithm. In this paper Bertekamp's mothod is applied to the VLSI desizn of a (255, 22: . RS-encoder, which can be implemented on a single VLSI chip.

\section{H. Borlokamp's Bh-Sertal Multiplisr Algorithm Over GF (2m)}

In order to understand Berlekamp's multiplier aigorithm some mathematical preliminaries are needed. Toward this end the mathematical concepts of the trace and a complementary (or dual) basis are introduced. For more details and proofs see Refs. 3, 4 and 5 .

Definition 1: The trace of an element $p$ belonging to $G F\left(p^{m}\right)$, the Galois field of $P^{m}$ elements, is defined as follows:

$$
\operatorname{Tr}(\beta)=\sum_{k=0}^{m-1} \beta^{k}
$$

In particular, for $p=$ ?

$$
M(\beta)=\sum_{k=0}^{m-1}(\beta)^{k}
$$

The trace has the following properties

1) $[M(\beta)]^{p}=\beta+\beta^{p} \cdot \cdots+\beta^{m-1}=\operatorname{Mr}(\beta)$. where $\beta \epsilon$ $G F\left(p^{m}\right)$. This implies that $T(\beta) \in G F(p)$ i.e.. the trace is on the ground field $G i(i)$

(2) $\operatorname{Tr}(\beta+r)=\operatorname{Tr}(\beta)+\operatorname{Mr}(r)$, where $\beta, r \in G F\left(p^{m}\right)$

(3) $T(c \beta)=c \operatorname{Tr}(\beta)$, where $c \in G F(p)$.

(4) $M(1) \equiv \operatorname{mon}(\bmod p)$

L.jumtion 2: A basss $(\mu$,$\} in G F\left(p^{m}\right)$ is a set of $m$ linearly independent elements in $G F\left(p^{m}\right)$.

Definition 3: Two bases $\left\{\mu_{j} ;\right.$ and $\left\{\lambda_{k}\right\}$ are said to be complementary or the dual of one another if

$$
\operatorname{Mr}\left(\mu_{j}\right)=\left\{\begin{array}{l}
1, \ddot{a} ;=k \\
0 . j \neq k
\end{array}\right.
$$


The basis $\left\{\mu_{t}\right\}$ is called the original basis, and the basis $\left\{\lambda_{k}\right\}$ is callod the dual basis.

Lemma: If $\alpha$ is a root of an irreducible pabnomial of degree $m$ in $G F\left(p^{m}\right)$, then $\left\{\alpha^{k}\right\}$ for $0<k<m-1$ is a basis of $G F\left(b^{m}\right)$. The basis ( $a^{k}$ ) for $0<k<m-1$ is called the normal or natural basis of $G F\left(p^{m}\right)$.

Theonem I (Theorem 19 in Ref. 4): Every basis has a complementary basis.

Covollary 1: Supposes the bases $\left\{\mu\right.$, and $\left\{\lambda_{k}\right\}$ are complementary. Then a field element $z$ can be expressed in the dual basis $\left\{\lambda_{k}\right\}$ by the expansion

$$
z=\sum_{k=0}^{m-1} z_{k} \lambda_{k}=\sum_{k=0}^{m-1} M\left(2 \mu_{k}\right) \lambda_{k}
$$

where $z_{k}=T\left(z \mu_{k}\right)$ is the $k$ th coefficient of the dual bicsis.

Proof: Let $z=z_{0} \lambda_{0}+z_{1} \lambda_{1}, \cdots+z_{m-1} \lambda_{m-1}$. Multiply both sides by $a^{k}$ and take the trace. Then by Def. 3 and the properties of the trace.

$$
\operatorname{rr}\left(z \alpha^{k}\right)=\operatorname{rr}\left(\sum_{i=0}^{m-1} z_{i}\left(\lambda_{i} \mu_{k}\right)\right)=z_{k} \quad \text { Q.E.D. }
$$

The tollowing cor llary is an immediate consequence of Corollary 1.

Conollary 2: The product $w=z y$ of two tigeld elements in $G F\left(p^{m}\right)$ can be expressed in the dual basis by the expansion

$$
n \cdot=\sum_{k=0}^{m-1} T\left(z y \mu_{k}\right) \lambda_{k}
$$

where $T H\left(z y \mu_{k}\right)$ is the $k$ th coefficient of the dual basis for the product of two hield elcments.

These two corollaries provide a theoretical basis for the new RS-encuder algonthm.

\section{A Simple Example of Berlekamp's Algorithm Applied to an RS-Encoder}

This section follows the treatment in Ret. 3 . It is included here for two purposes. First, $\operatorname{Ref} .3$ is not readily -vailable for most readers. Second. this example is incluised to illusirate how Borieknmp's new biterial multiplis aforithm an be used to realize the RS-encoder structure presented ia Fis. 1.

Consider a (15, 11) RS cod. over $G F\left(2^{4}\right)$ For th: cods, $m$ $=4, n=15, t=2, d=2 t+1=5$, and $n-2 t=1 !$ information symbols. Let a be $a$ root of the primitive irroductole polynomial $f(x)=x^{4}+x+1$ over GP(2). a atisfies $a^{15}=1$. An element $z$ in $G F\left(2^{4}\right)$ is representable by $00: \alpha$ for some $j$, $0<j<14.2$ can be represented also by a polynominl in a over $G F(2)$. This is the representation of $G F\left(2^{\circ}\right)$ in the normal basis $\left(\alpha^{k}\right)$ for $0<k<3$. That is, $z=u_{0}+u_{1} \alpha+u_{2} \alpha^{2}+u_{3} \alpha^{3}$, where $u_{k} \in G F(2)$ for $0<k<3$.

In Table 1, the first column is the index or logarithm of an element in base $a$. The logrithm of the zero element is denotza by an asterisk. Columans 2 to 5 show the 4-tuples of the coefficients of the elements expressed as polynomials.

The trace of an element $z$ in $G F\left(2^{4}\right)$ is found by $2 e f .1$ and the properties of the trace to be

$$
\operatorname{TR}(z)=u_{0} \operatorname{Mr}(1)+u_{1} \operatorname{Tr}(a)+u_{2} \operatorname{Tr}\left(\alpha^{2}\right)+u_{3} \operatorname{Tr}\left(\alpha^{3}\right)
$$

where $\operatorname{Tr}(1) \equiv 4(\bmod 2)=0, \operatorname{Tr}(a)=\operatorname{Tr}\left(a^{2}\right)=a+a^{2}+a^{4}+$ $a^{8}=0$ and $\operatorname{Tr}\left(\alpha^{3}\right)=a^{3}+a^{6}+a^{2}+a^{12}=1$. Thus $\operatorname{Tr}(z)=u_{3}$. The trace element $\alpha^{*}$ in $G F\left(2^{4}\right)$ is listed in column 3 of Table 1 .

By Def. 2 any set c tour linearly independent elements can be used as a basis for the field $G F\left(2^{4}\right)$. To find the dual basis of he normal basis $(d)$ in $G F\left(2^{4}\right)$ let a field element $z$ be expressed in dual basis $\left\{\lambda_{0}, \lambda_{1}, \lambda_{2}, \lambda_{3}\right\}$. From Corollary 1 the coefficients of $z$ are $z_{k}=\operatorname{Tr}\left(z \alpha^{k}\right)$ for $0 \leqslant k<3$. Thus $z_{0}=$ $\operatorname{Tr}(z), z_{1}=\operatorname{Tr}(z a), z_{2}=\operatorname{Tr}\left(z \alpha^{2}\right)$ and $z_{3}=\operatorname{Tr}\left(z a^{3}\right)$. Let $z=\alpha$ for some $i, 0 \leqslant i \leqslant 14$. Thus a coefficient $z_{k}$, for $0 \leqslant k \leqslant 3$, of an element $z$ in the dual space can be obtained by cyclically shifting the trace column in Table I upward by' $k$ positions where the first row is excluded. These appropristely shifted columns of coefficients are shown in Table 1 as the last four columns. In Table 1 the elements of the dual basis, $\lambda_{0}, \lambda_{1}, \lambda_{2}$. $\lambda_{3}$, are un.terlined. Evidently $\lambda_{0}=a^{14}, \lambda_{1}=a^{2}, \lambda_{2}=\alpha$ and $\lambda_{3}$ $=1$ are the four elements of the dual basis.

In order to make the generator polynomial $g(x)$ symmetric $b$ must satisfy the equation $2 b+d-2=2^{m}-1$. Thus $b=6$ for this code. The $\gamma$ in Eq. (2) can be any primitive el ment in $G F\left(.^{4}\right)$. It will be shown in Section IV that $\gamma$ can be chosen to simplify the binary mapping matrix. In this exainple let $\gamma=\alpha$. Thus the generator polynomial is given by

$$
g(x)=\prod_{i=6}^{9}\left(x-a^{j}\right)=\sum_{i=0}^{4} \varepsilon_{1} x^{i}
$$




\section{ORIGNAL FNE
OF POOR QUALTY}

where $g_{0}=g_{4}=1, g_{1}=g_{3}=\alpha^{3}$ and $g_{2}=\alpha$.

Let $g$, be expressed in the normal basis $\left\{1, a, \alpha^{2}, a^{3}\right\}$. Let 2 , a field element, be expressed in the dual basis; i.e., $z=z_{0} \lambda_{0}+$ $z_{1} \lambda_{1}+z_{2} \lambda_{2}+z_{3} \lambda_{3}$. In Fis. 1 the products $z_{i}$ for $0<i<3$ needs to he compited.

Since $g_{3}=g_{1}$, it it aecessary to compute only $2 g_{0}, 2 g_{1}$ and $2 g_{2}$. Let the products $2 g_{1}$ for $0 \leqslant i \leqslant 2$ be represented in the dual basis. By Corrollary $2 z$, can be expressed in the dual basis as

$$
z\left[\begin{array}{l}
g_{0} \\
g_{1} \\
g_{2}
\end{array}\right]=\sum_{k=0}^{3}\left[\begin{array}{ll}
T_{0}^{(k)} & (z) \\
T_{1}^{(k)} & (z) \\
T_{2}^{(k)} & (z)
\end{array}\right] \lambda_{k}
$$

where $T^{(k)}(z)=\operatorname{Tr}\left(2 g_{i} a^{k}\right)$ is the $k$ th coefficient (or $k$ th bit) of $z g_{i}$ for $0 \leqslant i \leqslant 2$ and $0 \leqslant k \leqslant 3$.

The present problem is to express $T_{i}^{(k)}(z)$ recursively in terms of $T_{i}^{(k-1)}(z)$ for $1 \leqslant k \leqslant 3$. Initially for $k=0$,

$$
\left[\begin{array}{l}
T_{0}^{(0)}(z) \\
T_{1}^{(0)}(z) \\
T_{2}^{(0)}(z)
\end{array}\right]=\left[\begin{array}{l}
\operatorname{Tr}\left(z g_{0}\right) \\
\operatorname{Tr}\left(z g_{1}\right) \\
\operatorname{Tr}\left(z g_{2}\right)
\end{array}\right]=\left[\begin{array}{l}
\operatorname{Tr}\left(z \alpha^{0}\right) \\
\operatorname{Tr}\left(2 \alpha^{3}\right) \\
\operatorname{Tr}(z \alpha)
\end{array}\right]=\left[\begin{array}{l}
z_{u} \\
z_{3} \\
z_{1}
\end{array}\right]
$$

whe:e $\operatorname{TR}\left(z \alpha^{j}\right)=\operatorname{Tr}\left(\left(z_{0} \lambda_{0}+z_{1} \lambda_{1}+z_{2} \lambda_{2}+z_{3} \lambda_{3}\right) \alpha^{j}\right)=z_{i}$ for 0 $\leqslant j \leqslant 3$. Equation (9) can be expressed in a matrix form as follows:

$$
\left[\begin{array}{l}
T_{0}^{(0)}(z) \\
T_{1}^{(0)}(z) \\
T_{2}^{(0)}(z)
\end{array}\right]=\left[\begin{array}{llll}
1 & 0 & 0 & 0 \\
0 & 0 & 0 & 1 \\
0 & 1 & 0 & 0
\end{array}\right]\left[\begin{array}{l}
z_{0} \\
z_{1} \\
z_{2} \\
z_{3}
\end{array}\right]
$$

The above matrix is the $3 \times 4$ binary mapping matrix of the problem.
To compute $T^{(k)}(z)$ for $k>0$, observe that $T_{i}^{(k)}(z)=$ $M(\alpha z) a^{\left.\alpha^{k-1}\right)}=T^{(k-1)}(\alpha z)$. Hence $T^{(k)}(z)$ is obtrineo from $T_{i}^{(k-1)}(z)$ by replacing $z$ by $y=0$. Let ez $=y=y_{0} \lambda_{0}+y_{1} \lambda_{1}$ $+y_{2} \lambda_{3}+y_{3} \lambda_{1}$, where $y_{m}=M\left(y \alpha^{m}\right)=M\left(2 a^{m+1}\right)$ for $0<m$ $<3^{2}$. Then $T^{k}\left({ }^{k}\right.$ is obtained from $T_{i}^{(k-1)}$ by replectinz $z_{0}$ by $y_{0}$ $=\operatorname{Tr}(z a)=z_{1}, z_{1}$ by $y_{1}=\pi\left(z a^{2}\right)=z_{2}, z_{2}$ by $y_{2}=\pi\left(2 a^{3}\right)=z_{3}$ and $z_{3}$ by $y_{3}=M\left(z \alpha^{4}\right)=T(z(\alpha+1))=z_{0}+z_{1}$.

To recapitulate $2 g_{i}=T T^{(0)} \lambda_{0}+T \gamma^{(1)} \lambda_{1}+T^{(2)} \lambda_{2}+T_{i}^{(3)} \lambda_{3}$, where $0 \leqslant i<3$ and $z=z_{0} \lambda_{0}+z_{1} \lambda_{1}+z_{2} \lambda_{2}+z_{3} \lambda_{3}$, can be computed by Berlekamp's bit-serial multiplier algorithm as follows:

(1) Initially for $k=0$, compute $T_{0}^{(0)}(z), T_{1}^{(0)}(2)$ and $\Gamma_{2}^{(0)}(z)$ by Eq. (10). Also $T_{3}^{(0)}(2)=T_{1}^{(0)}(2)$.

(2) For $k=1,2,3$, compute $T_{i}^{(k)}(z)$ by

$$
T_{i}^{(k)}(z)=T_{i}^{(k-1)}(y)
$$

where $0<i<3$ and $y=\alpha z=y_{0} \lambda_{0}+y_{1} \lambda_{1}+y_{2} \lambda_{2}+$ $y_{3} \lambda_{3}$ with $y_{0}=z_{1}, y_{1}=z_{2}, y_{2}=z_{3}$ and $y_{3}=z_{0}+z_{1}=$ $T_{f}$ where $T_{f}=z_{0}+z_{1}$ is the feedback term of the algorithm.

The above example illustrates Berlekamp's bit-serial multiplier algorithm. This algorithm developed in Refs. 4 and 5 requires shifting and XOR operations only. Brylekamp : dual basis RS-encoder is well-suited to a pipeline structure which can be implemented in VLSI design. The same procedure extends similarly to the design of a $(255,223)$ RS-encoder uver $G F\left(2^{8}\right)$.

\section{A VLS1 Architecture of a $(255,223)$ RS-Encoder with Dual-Besis Multiplier}

In this section an architecture is designed to implement $(255,223)$ RS-encoder using Berlekamp's multiplier algorithm. The circuit is a direct mapping from an encoder using Berlekamp's bit-serial algorithm as developed in the previour sections to an architectural design. This architecture can be realized quite regdily on a single NMOS VLSI chip.

Let $G F\left(2^{8}\right)$ be generated by $\alpha$, where $a$ is a root of a primitive irreducible polyromial $f(x)=x^{8}+x^{7}+x^{2}+x+1$ over $G F(2)$. The nurmal basis of this field is $\left\{1, \alpha, \alpha^{2}, a^{3}, a^{4}\right.$, $\left.a^{5}, a^{6}, a^{7}\right\}$. The representations of this field in both the normal basis and its dual basis are tabulated in Appendix A. Frum Corrollary 1 the cuefficients of a field element $\alpha$ can be obtained from $z_{k}=\operatorname{Tr}\left(\alpha^{\alpha+k}\right)$ for $0<k<7$, where $\alpha=z_{0} \lambda_{0}$ $+\cdots+z_{7} \lambda$. From Table A-1 in Appendix A, the dual basis 
$\left\{\lambda_{0}, \lambda_{1}, \cdots, \lambda_{3}\right\}$ of the normal basis is the ordered set $1 a^{99}$, $a^{187}, a^{203}, a^{202}, a^{201}, a^{200}, a^{199}, a^{100}$.

It was mentioned previously that $\gamma$ in Eq. (2) can be chosen to siniplify the binary mapping matrix. Two binary matrices. one for the primitive element $\gamma=a^{\prime \prime}$ and the other for $\gamma=a$, were computed. It was found that the binary mapping matrix for $\gamma=a^{11}$ had a smaller number of 1 's. Hence this binary mapning matrix was used in the design. For this case the generator polynomial $g(x)$ of the RS-encoder over $G F\left(2^{6}\right)$ was given by

$$
g(x)=\prod_{i=112}^{143}\left(x-a^{11 j}\right)=\sum_{i=0}^{32} g_{i} x^{i}
$$

where $g_{0}=g_{32}=1, g_{1}=g_{31}=\alpha^{249} \cdot g_{2}=g_{30}=\alpha^{59} \cdot g_{3}=g_{29}$ $=a^{66} \cdot g_{4}=g_{28}=a^{4} \cdot g_{5}=g_{27}=a^{43} \cdot g_{6}=g_{26}=a^{126} \cdot g_{7}=$ $g_{25}=\alpha^{251} \cdot g_{8}=g_{24}=\alpha^{97} \cdot g_{9}=g_{23}=\alpha^{30} \cdot g_{10}=g_{22}=\alpha^{3}$. $g_{11}=g_{21}=\alpha^{2 \cdots} g_{12}=g_{20}=\alpha^{50} \cdot g_{13}=g_{19}=\alpha^{66} \cdot g_{14}=$ $g_{18}=\alpha^{170}, g_{14} \quad, 17=\alpha^{5}$, and $g_{16}=\alpha^{24}$

The binary napping matrix for the coefficients of the generator poly $1 \mathrm{mial}$ in Eq. (11) is computed and shown in Appendix B. $T$ : feedback term $T_{f}$ in Beriekamp's algorithm is found in this , ise to be:

$T_{f}=\operatorname{Tr}\left(\alpha^{8} z\right)=\operatorname{Tr}\left(\left(\alpha^{7}+\alpha^{2}+\alpha+1\right) z\right)=z_{0}+z_{1}+z_{2}+z_{7}$

In the following a VLSI thip architecture is designed to realize a $(255,223)$ RS-encoder using the above parameters and Berlekamp's algorithms. An overall block diagram of this chip is shown in Fig. 2. In Fig. 2 VDD and GND are power pirs. CLK is a cinik signal, which in general is a periodic square wave. The information symbols are fed into the chip from the data-in pin DIN bit-by-bit. Similarly the encoded codeword is transmitted out of the chip fr $n$ t'le data-out pin DOUT sequentially. The control signal LM (loac nitode) is set to 1 (logic 1) when the information symbols are loaded into the chip. Otherwise, LM is set to 0.

The input data and LM signals are synchronized by the CLK signal. while the operations of the circuit and output data signal are synchronized by two nunoverlapping clock signals

$$
\begin{aligned}
& \text { ra re ace. dynamic registers are used in this } \\
& \because \quad \text { of a l-bit dynanic register with reset is } \\
& \because \text { " "ng diagram of CLK, } \$ 1,02, L M, \text { DIN } \\
& \text { "m " : } \text { " Fig. 4. The delay of DOUT } \\
& \because \quad \quad \therefore . ., \quad \text {, the input and output flin-flops. }
\end{aligned}
$$

Fiqure 5 shows the block diagram of a (255, 223) RS-encuder over $G F\left(2^{8}\right)$ using Berlekamp's bit-serial multiplier algorithm. The circuit is divided into five units. The circuits in each unit are discussed in the following:

(1) Product Unit: The Product Unit is used to compute $T_{\rho}$ $T_{31}, \cdots, T_{0}$. This circuit is realized by a Programmable Logic Array (PLA) circuit [6]. Since $T_{0}=T_{31}$. $T_{1}=T_{30}, \cdots, T_{15}=T_{17}$, only $T_{p} T_{31}, \cdots, T_{17}$ and $T_{16}$ are actually implemented in the PLA circuit. $T_{0}, \cdots, T_{15}$ are connected directly to $T_{31}, \cdots, T_{17}$, respectively. Over other circuits a PLA circuit has the advantage of being easy to recn...izure.

(2) Remainder Unit: The Remainder Unit is used to store the coefficients of the remainder during the diricion process In Fig. $5, S_{i}$ for $0 \leqslant i \leqslant 3 n$ are 8-tit shift registers with reset. The addition in the circuit is a modulo 2 addition or Exclusive-OR operation. While $c_{32}$ is being fed to the circuit, $c_{31}$ is being computed and iransmitted sequentially from the wrcuit. Simul taneously $c_{i}$ is computed and then loaded into $S_{i}$ for $0 \leqslant i \leqslant 30$. Then $c_{30}, \cdots, c_{0}$ are transm::ted out of the encoder bit-by-bit.

(3) Quotient Unit: In Fig. 5, $Q$ and $R$ represent a 7 bit shift register with reset ard an 8-bit shift register with reset and parallel load, respectively. $R$ and $Q$ store the currently operating coefficient and the next coefficient of the quotient polynomial, respectively. A logic diagram of register $R$ is shown in Fig. 6. $z_{i}$ is loaded into $R$ ever; eight clock cycles, where $0 \leqslant i \leqslant 7$. Immeduately after all 223 information symbols are fed intc the circuit, the control signal SL changes to logic 0 . Thenseforth the contents of $Q$ and $R$ are zerc so that the check symbols in the Remainder Unit sustain their values.

(4) 1/0 Unit: This unit handles the input/output operations. In Fiz. 5 both $F_{0}$ anc $F_{\text {, }}$ are flip-flops. $A$ pass transistor controlled by $\phi 1$ is inserted before $F$ for the purpose of synchronization. Control signal SL selects wirether a bit of an information symbol or a check symbol is to be transmitted.

(5) Control Unit: The Control Unit generates the necessary control signals. This unit is further divided into , portions, as shown in Fig. 7. The two-phase clock generator circuit in Ref. 6 is used to convert a clock signal intc two nonoverlapping clock signals $\phi 1$ and $\phi 2$. In Fig. 8 is shown a logic diagram of the circuit for generating control signals START and SL. Control signal START resets all registers and the divide-by-8 
counter before the encoding process begins. Contral signal $L D$ is simply generated by a divido-by -8 counter to load the $z_{j}$ 's into the $R_{j}$ 's in parallel.

ince a codeword contains 255 symbols, the computation of a camplete encoded codeword requires 355 "symbol sycles." A symbol cycle is the time interval for execting a complete cycle of Berlekamp's algorithm. Since a symbol has 8 bits, a symbol cycle contains 8 "bit cycles." A bit cycle is the time interval for executing one step in Berlekamp's algorithm. In this design a bit cycle requires a period of the clock cycle.

The layout design of this $(255,223)$ RS-encoder is shown in Fig. 9. Before the design of the layout each circuit was simulated on a general-purpose computcr by using SPICE (a transistortevel circuit simulation program) (Ref. 7). The cir- crit requires about 3000 transistors, while a similar JPL destan requires 30 CMOS IC chips (Ref. 5). This RS-encoder detign will be fabricated and tested in the near future.

\section{Concluding Remarks}

A VISI structure is developed for a Reed-Salomon encoder using Berlekamp's bit-serial multiplier algorithm. This structure is both regular and simple.

The circuit in Fig. 2 can be modified easily to encode an RS code with a different field representation and different parameters other than those used in Section IV. Table 2 shows the primary modifications needed in the circuit to change a given parameter.

\section{Acknowledgment}

The authors wish to acknowledge Marvin Perlman of the Information Systems Engineering Section of JPL. His initial work with $E$. Berlekamp eventually resulted in the acceptance of the Berlekamp code as a guideline for both NASA and ESA. Also, his tutorial paper on the algorithm (Refarence 5) was particularly helpful to the authors.

\section{References}

1. Reefs, H. F. A.. and Best. A. R.. "Concatenated Coding on A Spacecraft-10-Ground Telemetry Channel Pe:Surmance," Proc. ICC 81, Denver, 1981.

2. Odenwader. J., et al., "Hybrid Coding System Study Final Report," Linkabit Corp., NASA CRI14, 486, September 1972.

3. MacWilliams. P. J., and Slcane. N.J.A., The Theon Of Error-Correcting Codes, Nurth-Holand Publishirig Cur. i, ?v. 1978

4. Berlekanip. E. R.. "Techr. Proposal for A Low-Power Reed-Solomon Encoder/ Interleaver Using About $30 \mathrm{C}_{1 .} \mathrm{IOS}$ IC's," Cyclotomics, Inc., in response to RFP No. BP-6-9007.

5. Periman. M. anc Lee, J. J., "A Comparison of Conventional Reed-Solomon Encoders and Berlekamp's Architecture," New Technology Repont NPO-15568, March 10, 1982. Case Nu. D-15568. NASA.

6. Mead, C., and Conway, L. Introduction To VLSI Systems, Addison-Wesley Publishing Company, Calif., 1980.

7. Negal, L. W., and Pederson, D. O.. "SPICE - Simulation Program with Integrated Cir. c'llt Emphasis," Memoranduni No. ERL-in302. Electronics Research Laboratory. University of Calıfornı, Berkelev. April 12. 1973. 


\section{original page n \\ OF POOR QUALITY}

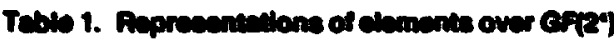

conarited by $a^{4}=a+1$

\begin{tabular}{|c|c|c|c|}
\hline $\begin{array}{c}\text { Power } \\
j\end{array}$ & $\begin{array}{l}\text { Elements in } \\
\text { normal base }\end{array}$ & $\boldsymbol{r}\left(\boldsymbol{a}^{j}\right)$ & $\begin{array}{l}\text { Elements in } \\
\text { dual bese }\end{array}$ \\
\hline & $\alpha^{3} a^{2} a^{1} a^{0}$ & & $x_{0}^{2} 1_{2}^{2}{ }_{3}$ \\
\hline * & 0000 & 0 & 0000 \\
\hline 0 & 0001 & 0 & $0001 \lambda_{3}$ \\
\hline 1 & 0010 & 0 & $\overrightarrow{0010 \lambda_{2}}$ \\
\hline 2 & 0100 & 0 & $\overline{0100 \lambda}$ \\
\hline 3 & 1000 & 1 & $\overrightarrow{1001}$ \\
\hline 4 & 0011 & 0 & 0011 \\
\hline 5 & 0110 & 0 & 0110 \\
\hline 6 & 1100 & 1 & 1101 \\
\hline 7 & 1011 & 1 & 1010 \\
\hline 8 & 0101 & 0 & 0101 \\
\hline 9 & 1010 & 1 & 1011 \\
\hline 10 & 0111 & 0 & 0111 \\
\hline 11 & 1110 & 1 & 1111 \\
\hline 12 & 1111 & 1 & 1110 \\
\hline 13 & 1101 & 1 & 1100 \\
\hline 14 & 1001 & 1 & $1000 \lambda_{0}$ \\
\hline
\end{tabular}

Table 2. The primary modilications of the encoder circult in Fig. 2 needed to change a paremeter

\begin{tabular}{|c|c|c|c|c|}
\hline & $\begin{array}{l}\text { Parameter } \\
\text { o be changed }\end{array}$ & $\begin{array}{l}\text { The value } \\
\text { used for } \\
\text { the cucuit } \\
\text { in l te. ? }\end{array}$ & $\begin{array}{l}\text { Neu } \\
\text { vasue }\end{array}$ & $\begin{array}{l}\text { The cucuits of Fig. } 2 \\
\text { that require modification }\end{array}$ \\
\hline 1 & $\begin{array}{l}\text { Gencratur } \\
\text { poly nomial }\end{array}$ & Lq. (8) & $g(x)$ & $\begin{array}{l}\text { The PLA of the Product } \\
\text { Unit neads to be } \\
\text { changed }\end{array}$ \\
\hline 2. & $\begin{array}{l}\text { The finite } \\
\text { tueld used }\end{array}$ & $G F\left(2^{8}\right)$ & $G F\left(2^{m}\right)$ & $\begin{array}{l}\text { All regusters are } m \text { bit } \\
\text { resistors. except } Q \text { is a } \\
\text { i } m-1 \text {-bit repaster. A } \\
\text { divde-by-m counter is } \\
\text { used. (The generator } \\
\text { poly numbl may not be } \\
\text { changed.) }\end{array}$ \\
\hline 3 & $\begin{array}{l}\text { frro:- } \\
\text { curtectung } \\
\text { capability }\end{array}$ & 16 & $t$ & $\begin{array}{l}2 t-2 \text { shift registers are } \\
\text { required in the Re- } \\
\text { mander Unit. The } \\
\text { ge netator poly nomial is } \\
\text { also changed.) }\end{array}$ \\
\hline 4 & $\begin{array}{l}\text { vimbet of } \\
\text { inturmation } \\
\text { sy mbols }\end{array}$ & 223 & $k$ & $\begin{array}{l}\text { None is changed. sunce } \\
k \text { is implicitly contained } \\
\text { in the control signal LM }\end{array}$ \\
\hline
\end{tabular}


ORIGINAL PAGE IS
OF POOR QUALITY

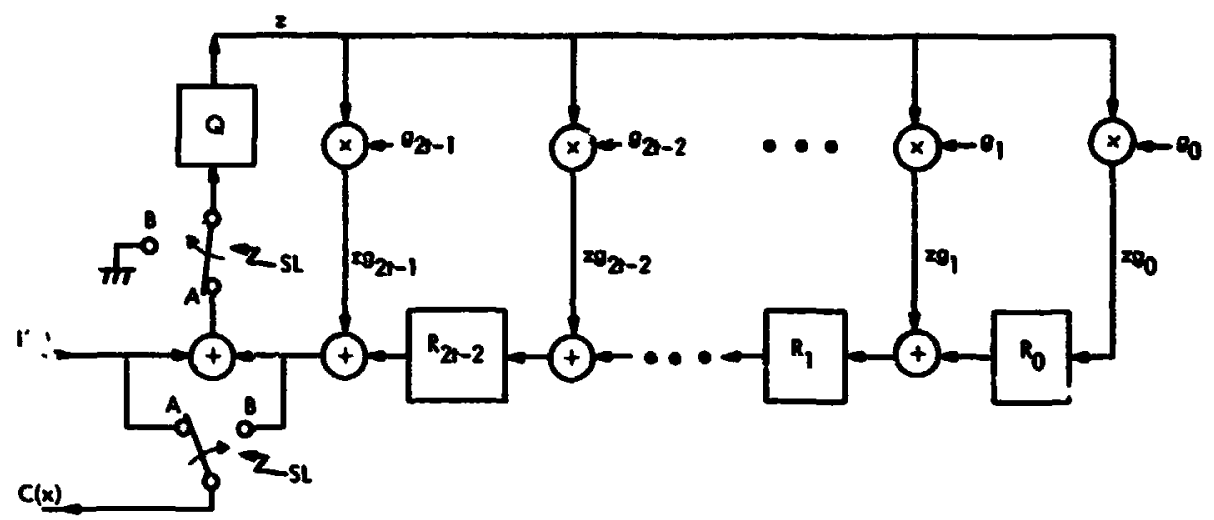

Fig. 1. A structure of a Rerror correting PB-encoder

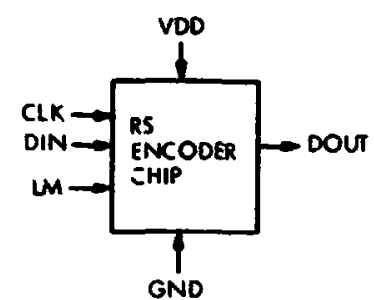

Fig. 2 Symbollc acoram of - Rs encoder chlp

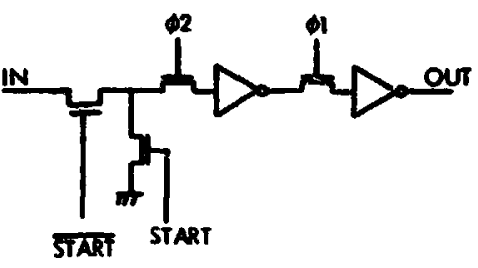

Fig. 3. Logic dearem of a 1th ofnamic reglater with recelt

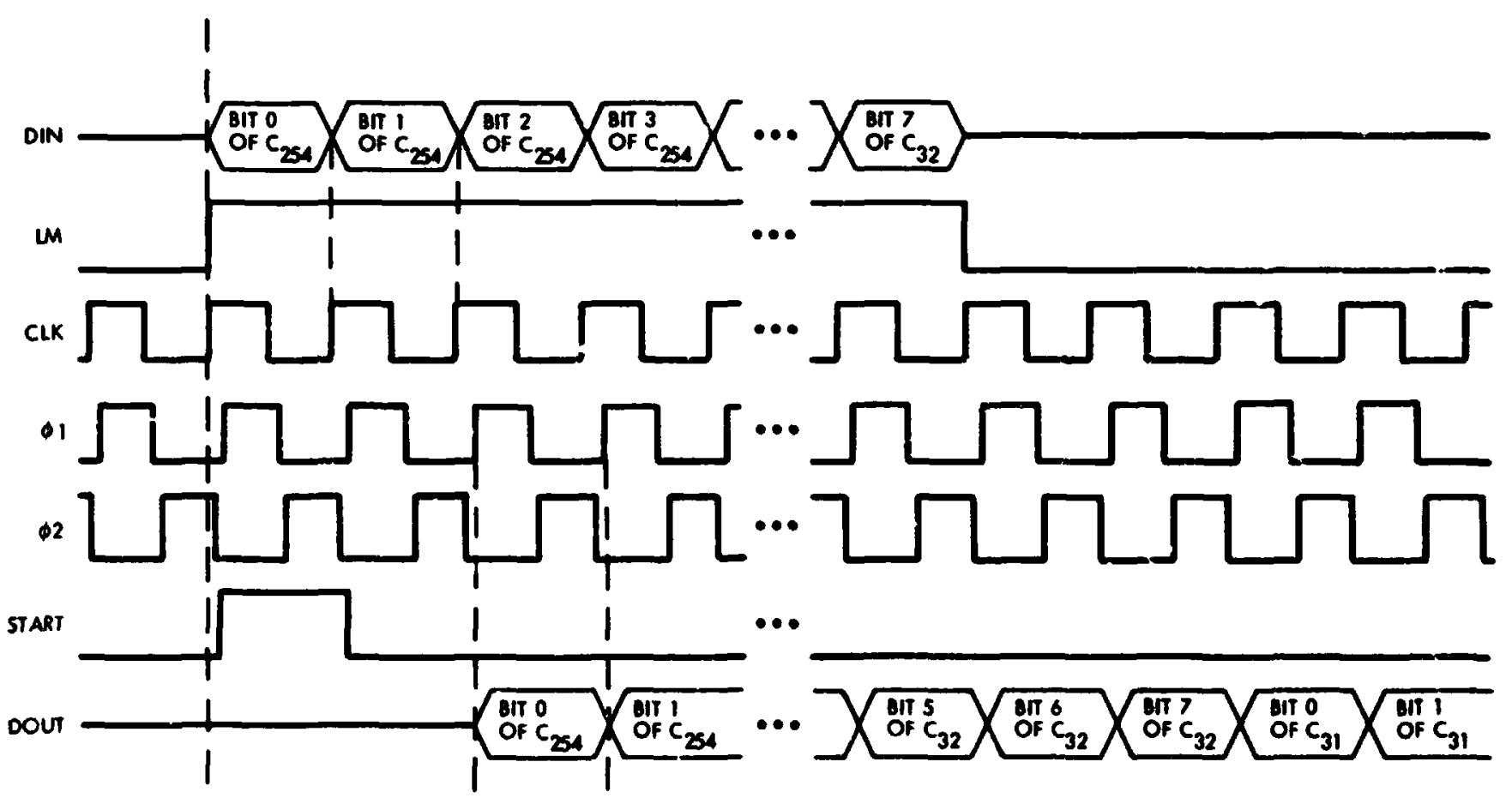

Fig. 4. The elgnals of DN, LM, CLK, \$1, \$2, 8TART, and OOUT 


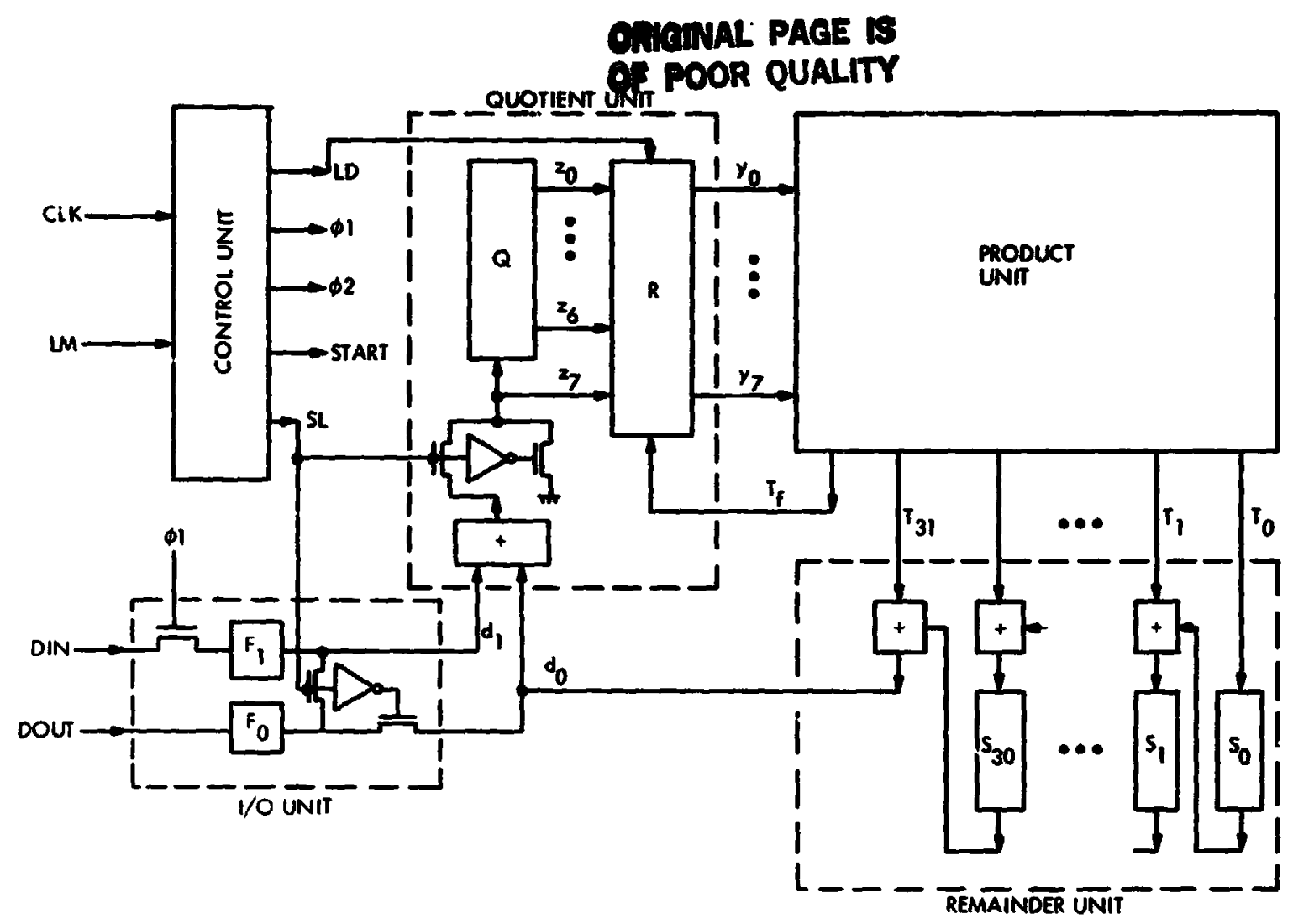

Fig. 5. Block dlagram of - . ancoder

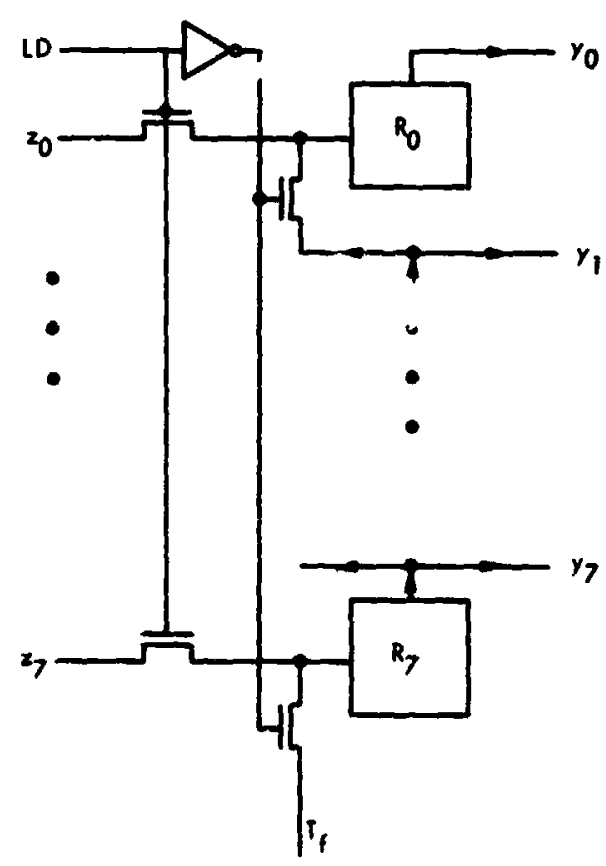

$R_{i}:$ A 1-BIT REGISTER WITH RESET

Fig. 6. Block dlagram of regioter $A$

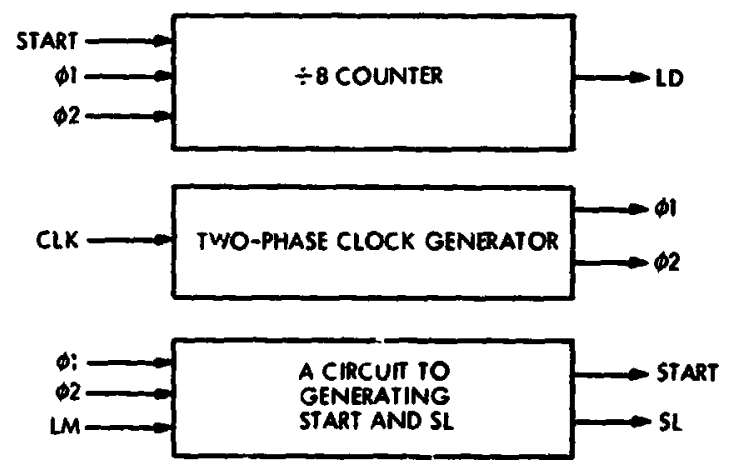

Flg. 7. Block disgram of the Control Unit

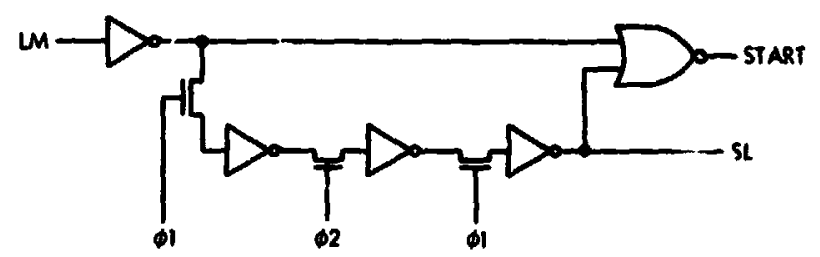

Fig. 8. Loglc diagram of the clicult for cenerating control signals START and 81 
ORIGINAL PAGE IS

OF POOR QUALITY



Fig, 9 Layout of the $(255,223)$ AS-enco otr chip 


\section{ORIGINAL PAGE: IS \\ OF POOR QUALITY}

\section{Appondix A}

In this appendix all 256 elements in $G F\left(2^{8}\right)$ are listed in Table A-1. These field elements are expressed in both the normal basis and its dual basis.

Teble A-i. Representations of clements in GF(?)

\begin{tabular}{|c|c|c|c|c|c|c|c|}
\hline $\begin{array}{c}\text { Power } \\
j\end{array}$ & $\begin{array}{c}\text { Elements } \\
\text { in nurina: } \\
\text { base }\end{array}$ & $\operatorname{Tr}\left(\alpha^{\prime}\right)$ & $\begin{array}{c}\text { Elements } \\
\text { in dual } \\
\text { base }\end{array}$ & $\begin{array}{c}\text { Power } \\
i\end{array}$ & $\begin{array}{c}\text { Elements } \\
\text { in normal } \\
\text { base }\end{array}$ & $\operatorname{Tr}\left(a^{\circ}\right)$ & $\begin{array}{c}\text { Elements } \\
\text { in dual } \\
\text { base }\end{array}$ \\
\hline - & 00000000 & 0 & 00000000 & 35 & 11101000 & 0 & 00110111 \\
\hline 0 & 00000001 & 0 & 01111111 & 36 & 01010111 & 0 & 01101110 \\
\hline 1 & 00000010 & 1 & 1111111 & 37 & 10101110 & 1 & 11011100 \\
\hline 2 & 00000100 & 1 & 11111110 & 38 & 11011011 & 1 & 10111000 \\
\hline 3 & $0000100 r$ & 1 & 11111101 & 39 & 00110001 & 0 & 01110000 \\
\hline 4 & 00010000 & 1 & 11111010 & 40 & 01100010 & 1 & 11100000 \\
\hline 5 & 00100000 & 1 & 11110101 & 41 & 11000100 & 1 & $: 1000001$ \\
\hline 6 & 01000000 & 1 & 11101010 & 42 & 00001111 & 1 & 10000011 \\
\hline 7 & 10000000 & 1 & 11010101 & 43 & 00011110 & 0 & 00000110 \\
\hline 8 & 10000111 & 1 & 10101011 & 44 & 00111100 & 0 & 00001100 \\
\hline 9 & 10001001 & 0 & 01010111 & 45 & 01111000 & 0 & 00011000 \\
\hline 10 & 10010101 & 1 & 10101110 & 46 & 11110000 & 0 & 00110000 \\
\hline 11 & 10101101 & 0 & 01011100 & 47 & 01100111 & 0 & 01100001 \\
\hline 12 & 11011101 & 1 & 10111001 & 48 & 11001110 & 1 & 11000011 \\
\hline 13 & 00111101 & 0 & 01110011 & 49 & 00911011 & 1 & 10000111 \\
\hline 14 & 01111010 & 1 & 11100111 & 50 & 00110110 & 0 & 00001110 \\
\hline 15 & 11110100 & 1 & 11001110 & 51 & 01101100 & o & 00011100 \\
\hline 16 & 01101111 & 1 & 10011100 & 52 & 11011000 & 0 & 00111000 \\
\hline 17 & 11011110 & 0 & 00111001 & 53 & 00110111 & 0 & 01110001 \\
\hline in & 00111011 & 0 & 01110010 & 54 & 01101110 & 1 & $111000_{1}$. \\
\hline 19 & 01110110 & 1 & 1110e'00 & 55 & 11011100 & 1 & 11000110 \\
\hline 20 & 1110.100 & 1 & 11001001 & 56 & 00111111 & 1 & 10001100 \\
\hline 21 & 01011111 & 1 & 10010011 & 57 & $0111 \cdot 110$ & 0 & 00011001 \\
\hline 22 & 10111110 & 0 & 00100110 & 58 & 11111100 & 0 & 00110011 \\
\hline 23 & 11111011 & 0 & 01001131 & 59 & 01111111 & 0 & 01100110 \\
\hline 24 & 01110001 & 1 & 10011010 & 60 & $11111 i 10$ & 1 & 11001100 \\
\hline 25 & 11100010 & 0 & 00110101 & 61 & 01111011 & 1 & 10011000 \\
\hline 26 & 01000011 & 0 & $01: 01010$ & 62 & 11110110 & 0 & 00110001 \\
\hline 27 & 10000110 & I & 11010100 & $6 ?$ & 01101011 & 0 & 01100010 \\
\hline 28 & 10001011 & I & 10101000 & 64 & 110110010 & 1 & 11000100 \\
\hline 29 & 10010001 & 0 & 01010000 & 65 & 00101011 & 1 & 10001000 \\
\hline 30 & 10100101 & 1 & $10110(x) 1$ & 66 & 01010110 & 0 & 00010001 \\
\hline 31 & 11001101 & 0 & $010000: 1$ & 67 & 10101100 & $\therefore$ & 00100011 \\
\hline 32 & 90011101 & 1 & 10000110 & 68 & 11011111 & 0 & 01000110 \\
\hline 33 & 00111010 & 0 & 00001101 & 69 & $00 \div .1001$ & 1 & 10001101 \\
\hline 34 & 01110100 & 0 & 00011011 & 70 & 01110010 & 0 & 00011010 \\
\hline
\end{tabular}


ORIGINAL PAGE IS
Thowa-1 (conta)

\begin{tabular}{|c|c|c|c|c|c|c|c|}
\hline $\begin{array}{c}\text { Power } \\
j\end{array}$ & $\begin{array}{l}\text { Elements } \\
\text { in normal } \\
\text { base }\end{array}$ & $\operatorname{Tr}\left(\alpha^{j}\right)$ & $\begin{array}{c}\text { Elements } \\
\text { in dual } \\
\text { base }\end{array}$ & $\begin{array}{c}\text { Power } \\
j\end{array}$ & $\begin{array}{c}\text { Elements } \\
\text { in normal } \\
\text { base }\end{array}$ & $\operatorname{Tr}\left(\alpha^{\prime}\right)$ & $\begin{array}{c}\text { Elements } \\
\text { in dual } \\
\text { base }\end{array}$ \\
\hline 71 & 11100100 & 0 & 00110100 & 112 & 01000111 & 1 & 10010100 \\
\hline 72 & r.001111 & 0 & 01101001 & 113 & $1 \sim 701110$ & 0 & $00101 C_{1}$ \\
\hline 73 & 10011110 & 1 & $11 C 10011$ & 114 & 10011011 & 0 & 01010010 \\
\hline 74 & 10111011 & 1 & 10100111 & 115 & 10110001 & 1 & 10100101 \\
\hline 75 & 11110001 & 0 & 01001111 & 116 & 11100101 & 0 & 01001011 \\
\hline 76 & 01100101 & 1 & 10011110 & 117 & 01001101 & 1 & 10010110 \\
\hline 77 & 11001010 & 0 & 00111101 & 118 & 10011010 & 0 & $0010110 \mathrm{i}$ \\
\hline 78 & 00010011 & 0 & 01111010 & 119 & 10110011 & & 01011010 \\
\hline 79 & 001001,0 & 1 & 11110100 & 120 & 11100001 & 1 & 10110101 \\
\hline 80 & $0100110 n$ & 1 & 11101001 & 121 & 01000101 & 0 & 01101011 \\
\hline 81 & 10011020 & 1 & 11010010 & 122 & 10001010 & 1 & .010111 \\
\hline 82 & 10110111 & 1 & 10100100 & $12 ;$ & 10010011 & 1 & 10101111 \\
\hline 83 & 11101001 & 0 & 01001000 & 124 & 10100001 & 0 & 01011111 \\
\hline 84 & 01010101 & 1 & 10010001 & 125 & 11000101 & 1 & 10111110 \\
\hline 85 & 10101010 & 0 & 00100010 & 126 & 00001101 & 0 & 01111100 \\
\hline 86 & $: 1010011$ & 0 & 01000101 & 127 & $000: 1010$ & 1 & 11111000 \\
\hline 87 & 00100001 & 1 & 10001010 & 128 & 00110100 & 1 & 11119001 \\
\hline 88 & 01000010 & 0 & 00010101 & 129 & 01101000 & 1 & 1110010 \\
\hline 89 & 10000100 & 0 & 00101011 & 130 & 11010000 & 1 & 11000101 \\
\hline 90 & 10001111 & 0 & 01010110 & 131 & 00100111 & 1 & ו1י 1000 \\
\hline 31 & 10011001 & 1 & 1010110! & 132 & 01001110 & 0 & 00010110 \\
\hline 92 & $10110 ; 01$ & 0 & 01011011 & 133 & $1001 \times 100$ & 0 & 00101100 \\
\hline y3 & 11101101 & 1 & ¿0110110 & 134 & 10111111 & o & 01011001 \\
\hline 94 & 01011101 & 0 & 01101100 & 135 & 11111001 & 1 & .0110010 \\
\hline 95 & 10111010 & 1 & 11011000 & 136 & 01110101 & 0 & 01100100 \\
\hline 96 & 11110011 & 1 & 10110000 & 137 & 11101010 & 1 & 11001000 \\
\hline$: 7$ & 01100001 & 0 & 01100000 & 138 & 01010011 & 1 & 10010000 \\
\hline 98 & 11000010 & 1 & 11000000 & 139 & 10100110 & 0 & 00100001 \\
\hline 99 & 00000011 & 1 & $10000000 \lambda_{0}$ & 140 & 11001011 & 0 & 01000010 \\
\hline 100 & 00000110 & 0 & $.20000001 \lambda_{7}$ & 141 & $00^{\prime} 310001$ & 1 & $100 m+1$ \\
\hline 101 & 00001100 & 0 & $\overline{00000011}$ & 142 & $0 J 140010$ & 0 & 00001010 \\
\hline 102 & 00011000 & 0 & $000 C 0111$ & 143 & 61000100 & 0 & $000 i 0100$ \\
\hline 103 & 00110000 & 0 & 00001111 & 144 & 10611400 & 0 & 00101000 \\
\hline 104 & 01100000 & 0 & 00011111 & $14 j$ & 10010111 & 0 & 01010001 \\
\hline 105 & 11000000 & 0 & c7111111 & 146 & 10101001 & 1 & 10100010 \\
\hline 106 & 0000111 & $r$ & 01111110 & $11^{17}$ & 11010101 & 0 & 01000100 \\
\hline 107 & on. 1110 & & 11111100 & 148 & 00101101 & 1 & 10001001 \\
\hline 108 & 00011100 & : & 11111001 & 149 & 01011010 & 0 & 00010010 \\
\hline 109 & 00111000 & 1 & 11110010 & 150 & $10^{\circ}: 0100$ & 0 & 00100100 \\
\hline 110 & 01110000 & 1 & 11100101 & isi & 11101111 & is & $01^{\prime \prime} 010 \mathrm{C}$ \\
\hline 111 & 11100000 & $:$ & 11001010 & 152 & $0101100 !$ & 1 & 10010010 \\
\hline
\end{tabular}


OKIGIVAL MULA
OF POOR QUALIT

Teble A-1 (contod)

\begin{tabular}{|c|c|c|c|c|c|c|c|}
\hline $\begin{array}{c}\text { Power } \\
j\end{array}$ & $\begin{array}{c}\text { Elements } \\
\text { in norm's } \\
\text { base }\end{array}$ & $\operatorname{Tr}\left(\alpha^{j}\right)$ & $\begin{array}{c}\text { Flements } \\
\text { in dual } \\
\text { base }\end{array}$ & $\begin{array}{c}\text { Power } \\
i\end{array}$ & $\begin{array}{c}\text { Elements } \\
\text { in normal } \\
\text { base }\end{array}$ & $\operatorname{Tr}(\alpha)$ & $\begin{array}{c}\text { Elements } \\
\text { in dual } \\
\text { base }\end{array}$ \\
\hline 153 & 10110010 & 0 & 00100101 & 194 & 01001001 & 0 & 01101000 \\
\hline 154 & 11100011 & 0 & 01001010 & 195 & 10010010 & 1 & 11010000 \\
\hline 155 & $01 * 2 n 001$ & 1 & 10019101 & 196 & 10100011 & 1 & 10100000 \\
\hline $1 \therefore$ & $\cdot .010$ & o & 00101010 & 197 & 11000001 & o & $01000000 \lambda_{1}$ \\
\hline 15 & $10 \mathrm{unt} 011$ & 0 & 01010101 & 198 & 00000101 & 1 & 10000001 \\
\hline 155 & 10000001 & 1 & 10101010 & 199 & 00001010 & $\mathbf{0}$ & $00000010 \lambda_{6}$ \\
\hline 159 & $10 \times 20101$ & 0 & 01010100 & 200 & 00010100 & 0 & $00000100 \lambda_{5}$ \\
\hline 160 & 10001101 & ' & 10101001 & 201 & 00101000 & o & $00001000 \lambda_{4}$ \\
\hline 161 & 10011101 & 0 & 01010011 & 202 & 01010000 & 0 & $\overline{00010000 \lambda_{3}}$ \\
\hline 162 & 10111101 & 1 & 10100110 & 203 & 10100000 & o & $\overline{00100000 \lambda_{2}}$ \\
\hline 163 & 11111101 & 0 & 01001100 & 204 & 11000111 & 0 & 01000001 \\
\hline 164 & 01111101 & 1 & 10011001 & 205 & 00001001 & 1 & 10000010 \\
\hline 165 & 11111010 & 0 & 00110010 & 206 & 00010010 & 0 & 00000101 \\
\hline 166 & 01110011 & o & 01100101 & 207 & 00100100 & 0 & 00001011 \\
\hline 167 & 11100110 & 1 & 11001011 & 208 & 01001000 & 0 & 00010111 \\
\hline 168 & 01001011 & 1 & 10010111 & 209 & 10010000 & 0 & 00101111 \\
\hline 169 & 10010110 & 0 & 00101110 & 210 & 10100111 & $\mathbf{0}$ & 01011110 \\
\hline 170 & 1010101: & 0 & 01011101 & 211 & 11001001 & 1 & 10111101 \\
\hline 171 & 11010001 & $\mathbf{l}$ & 10111010 & 212 & 00010101 & 0 & 01111011 \\
\hline 172 & 00100101 & o & 01110100 & 213 & 00101010 & 1 & 11110111 \\
\hline 173 & 01001010 & 1 & 11101000 & 214 & 01010100 & 1 & 11101110 \\
\hline 174 & 10010100 & 1 & 11010001 & 215 & 10101000 & 1 & 11011101 \\
\hline 175 & 10101111 & 1 & 10100011 & 216 & 11010111 & 1 & 10111011 \\
\hline 176 & 11011001 & 0 & 01000111 & 217 & 00101001 & 0 & 01110111 \\
\hline 177 & 00110101 & 1 & 10001110 & 218 & 01010010 & 1 & 11101111 \\
\hline 178 & 01101010 & o & 00011101 & 219 & 10100100 & 1 & 11011110 \\
\hline 179 & 11010100 & $\mathbf{0}$ & 00111011 & 220 & 11001111 & 1 & 10111100 \\
\hline 180 & 00101111 & 0 & 01110110 & 221 & 00011001 & 0 & 01111000 \\
\hline 181 & 01011110 & 1 & 11101100 & 222 & 00110010 & 1 & 11110020 \\
\hline 182 & 10111100 & 1 & 11011001 & 223 & 01100100 & 1 & 11100001 \\
\hline 183 & 11111111 & 1 & 10110011 & 224 & 11001000 & 1 & 11000010 \\
\hline 184 & 01111001 & 0 & 01100111 & 225 & 00010111 & 1 & 10000100 \\
\hline 185 & 11110010 & 1 & 11001111 & 226 & 00101 i 10 & 0 & 00001001 \\
\hline 186 & 01100011 & 1 & 10011111 & 227 & 01011100 & o & 00010011 \\
\hline 187 & 11000110 & 0 & 00111110 & 228 & 10111000 & o & 00100111 \\
\hline 188 & $000010^{\prime}$ & 0 & 01111101 & 229 & 11110111 & 0 & 01001110 \\
\hline 189 & 00010110 & 1 & 11111011 & 230 & 01101001 & 1 & 10011101 \\
\hline 190 & 00101100 & 1 & 11310110 & 231 & 11010010 & 0 & 00111010 \\
\hline 191 & 01011000 & 1 & 11101101 & 232 & 00100011 & 0 & $01110 i 01$ \\
\hline 192 & 10,10000 & 1 & 11011010 & 233 & 01000110 & 1 & $111010\}^{\prime}$ \\
\hline 193 & $\|I(x)\| \|$ & 1 & 10110100 & 234 & 100001100 & 1 & 11010110 \\
\hline
\end{tabular}


Toble a-1 (coned

\begin{tabular}{|c|c|c|c|c|c|c|c|}
\hline $\begin{array}{c}\text { Power } \\
i\end{array}$ & $\begin{array}{l}\text { Elements } \\
\text { in murmal } \\
\text { base }\end{array}$ & $\operatorname{Ir(\alpha ^{\prime })}$ & $\begin{array}{l}\text { Elements } \\
\text { in dual } \\
\text { base }\end{array}$ & $\begin{array}{c}\text { Power } \\
i\end{array}$ & $\begin{array}{c}\text { Elements } \\
\text { in normal } \\
\text { buse }\end{array}$ & $\operatorname{Tr}\left(\alpha^{j}\right)$ & $\begin{array}{c}\text { Sements } \\
\text { in dual } \\
\text { bre }\end{array}$ \\
\hline 235 & 10011111 & 1 & 10101100 & 245 & 01111100 & 1 & 11100110 \\
\hline 236 & 10111001 & 0 & 01011000 & 246 & 11111000 & 1 & 11001101 \\
\hline 237 & 11110101 & 1 & 10110001 & 247 & 01110111 & 1 & 10011011 \\
\hline 238 & 01101101 & 0 & 01100011 & 248 & 11101110 & 0 & 00110110 \\
\hline 239 & 11011010 & 1 & 11000111 & 249 & 01011011 & o & 01101101 \\
\hline 240 & 00110011 & 1 & 10001111 & 250 & 10110110 & 1 & 11011011 \\
\hline 241 & 01100110 & $\mathbf{0}$ & 00011110 & 251 & 11101011 & 1 & 10110111 \\
\hline 242 & 11001100 & 0 & 00111100 & 252 & 01010001 & 0 & 01101111 \\
\hline 243 & 00011111 & 0 & 01111001 & 253 & 10100010 & 1 & 1101111 \\
\hline 244 & 00111110 & 1 & 11110011 & 254 & 11000111 & $!$ & 1011111 \\
\hline
\end{tabular}




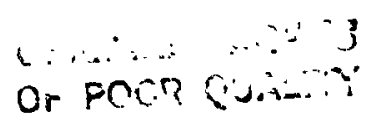

\section{Appendix $\mathrm{B}$}

The binary mapping matrix for $\gamma=\alpha^{11}$ of the $(255,223)$ RS-encoder is given by

$\left[\begin{array}{l}T_{0} \\ T_{1} \\ T_{2} \\ T_{3} \\ T_{4} \\ T_{5} \\ T_{6} \\ T_{7} \\ T_{8} \\ T_{9} \\ T_{10} \\ T_{11} \\ T_{12} \\ T_{13} \\ T_{14} \\ T_{15} \\ T_{16}\end{array}\right] \quad\left[\begin{array}{llllllll}1 & 0 & 0 & 0 & 0 & 0 & 0 & 0 \\ 1 & 1 & 0 & 1 & 1 & 0 & 1 & 0 \\ 1 & 1 & 1 & 1 & 1 & 1 & 1 & 0 \\ 0 & 1 & 1 & 0 & 1 & 0 & 1 & 0 \\ 0 & 0 & 0 & 0 & 1 & 0 & 0 & 0 \\ 1 & 0 & 1 & 1 & 0 & 0 & 0 & 0 \\ 1 & 1 & 0 & 1 & 0 & 1 & 1 & 1 \\ 1 & 0 & 0 & 0 & 0 & 1 & 1 & 0 \\ 1 & 0 & 1 & 0 & 0 & 1 & 0 & 1 \\ 0 & 0 & 0 & 1 & 0 & 0 & 0 & 0 \\ 0 & 1 & 0 & 1 & 0 & 1 & 0 & 0 \\ 0 & 1 & 1 & 0 & 1 & 1 & 0 & 0 \\ 0 & 1 & 1 & 0 & 1 & 0 & 1 & 0 \\ 1 & 1 & 0 & 1 & 0 & 1 & 0 & 1 \\ 0 & 0 & 0 & 0 & 0 & 1 & 0 & 0 \\ 1 & 0 & 0 & 0 & 1 & 1 & 1 & 0\end{array}\right]\left[\begin{array}{l}Z_{0} \\ Z_{1} \\ Z_{2} \\ Z_{3} \\ Z_{4} \\ Z_{5} \\ Z_{6} \\ Z_{7}\end{array}\right]$

